\title{
Ultraschall-Therapie bei Morbus Ménière
}

\begin{tabular}{|l|l|l|}
\hline B.N. & Reydon \\
\hline
\end{tabular}

Universiteit van Amsterdam, Keel-, Neus- en Oorheelkundige Kliniek (Hoofd: Prof. Dr. L. B. W. Jongkees)

\section{Adresse des Autors: Dr. B. N. Reydon, HNO Klinik, Universität von Amsterdam, Amsterdam (Holland)}

Es wurden zwei Jahre Erfahrung mit Ultraschall-Therapie an der Hals-, Nasen- und Ohrenklinik der Universität Amsterdam be-sprochen.

Obwohl es sich hier um em ziemlich kleines Material handelt, stimmen die Resultate ziemlich gut mit größeren Statistiken als denen von Altmann und Rüedí überein.

Wenn möglich wird die Operation unter Lokalanästhesie durchgeführt. Erstens, da die Art Nystagmus unser Leitfaden ist, zweitens in der Hoffnung, einer iatrogenen Fazialislähmung vorzubeugen. (Der einzige Fall mit einer postoperativen Fazialislähmung wurde unter allgemeiner Narkose operiert.)

Im Gegensatz zu Rüedí und Altmann bekamen wir in etwa der Hälfte der Fälle eine Gehörsverschlechterung an der operier-ten Seite. Unserer Meinung nach wird eine weitere Perfektionie-rung des Gerätes für die postoperativen Resultate günstig sein, die jetzt jedenfalls so sind, daß wir mit dieser Therapie weiterfahren, wenn auch nur in medikamentös-resistenten Fallen. 